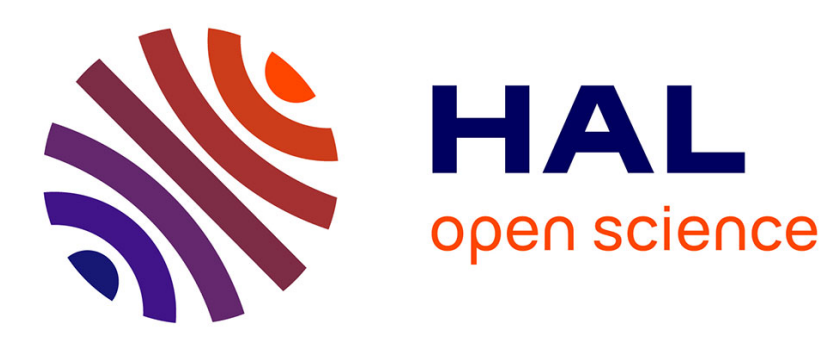

\title{
Inelastic electron holography - first results with surface plasmons
}

F. Röder, H. Lichte

\section{To cite this version:}

F. Röder, H. Lichte. Inelastic electron holography - first results with surface plasmons. European Physical Journal: Applied Physics, 2011, 54 (3), 10.1051/epjap/2010100378 . hal-00705149

\section{HAL Id: hal-00705149 \\ https://hal.science/hal-00705149}

Submitted on 7 Jun 2012

HAL is a multi-disciplinary open access archive for the deposit and dissemination of scientific research documents, whether they are published or not. The documents may come from teaching and research institutions in France or abroad, or from public or private research centers.
L'archive ouverte pluridisciplinaire HAL, est destinée au dépôt et à la diffusion de documents scientifiques de niveau recherche, publiés ou non, émanant des établissements d'enseignement et de recherche français ou étrangers, des laboratoires publics ou privés. 


\title{
Inelastic Electron holography - First results with surface plasmons
}

\author{
Falk Röder and Hannes Lichte \\ Triebenberg Laboratory, Institute of Structure Physics \\ Technische Universität Dresden \\ Dresden, Germany \\ www.triebenberg.de
}

\begin{abstract}
Inelastic interaction and wave optics seem to be incompatible in that inelastic processes destroy coherence, which is the fundamental requirement for holography. In special experiments it is shown that energy transfer larger than some $10^{-15} \mathrm{eV}$ undoubtedly destroys coherence of the inelastic electron with the elastic remainder. Consequently, the usual inelastic processes, such as phonon-, plasmon- or inner shell-excitations with energy transfer of several $\mathrm{meV}$ out to several $10 \mathrm{eV}$, certainly produce incoherence with the elastic ones. However, it turned out that within the inelastic wave, "newborn" by the inelastic process, there is a sufficiently wide area of coherence for generating "inelastic holograms". This is exploited to create holograms with electrons scattered at surface-plasmons, which opens up quantum mechanical investigation of these inelastic processes.
\end{abstract}

\section{Introduction: Inelastic Interaction, EELS and Holography}

For decades, Christian Colliex and his coworkers very successfully explored the basics of inelastic interaction and developed methods exploiting them for powerful materials analysis in the (S)TEM. As a new fascinating contribution to the fields, recently, they published energy-filtered images of triangularly shaped Ag-nanoparticles recorded in the light of the surface plasmons energy losses, which show striking eigenmode-like features (fig. 1) [1]. These images clearly indicate the structure of probability for excitation of such plasmons. The question is whether an exciting beam electron just experiences the excitation probability locally given by the nanoparticle, or "sees" the whole shape of the nanoparticle; in the latter case, the pattern would be a property also of the exciting beam electron. If it sees the whole particle, just as it "sees" both slits in a double-slit experiment, then there should be some interference possible between different areas of the excitation probability distribution in the nanoparticle. This is what we are striving for. As a prerequisite for this goal, we report about first holograms recorded with surface plasmon scattered electrons. 

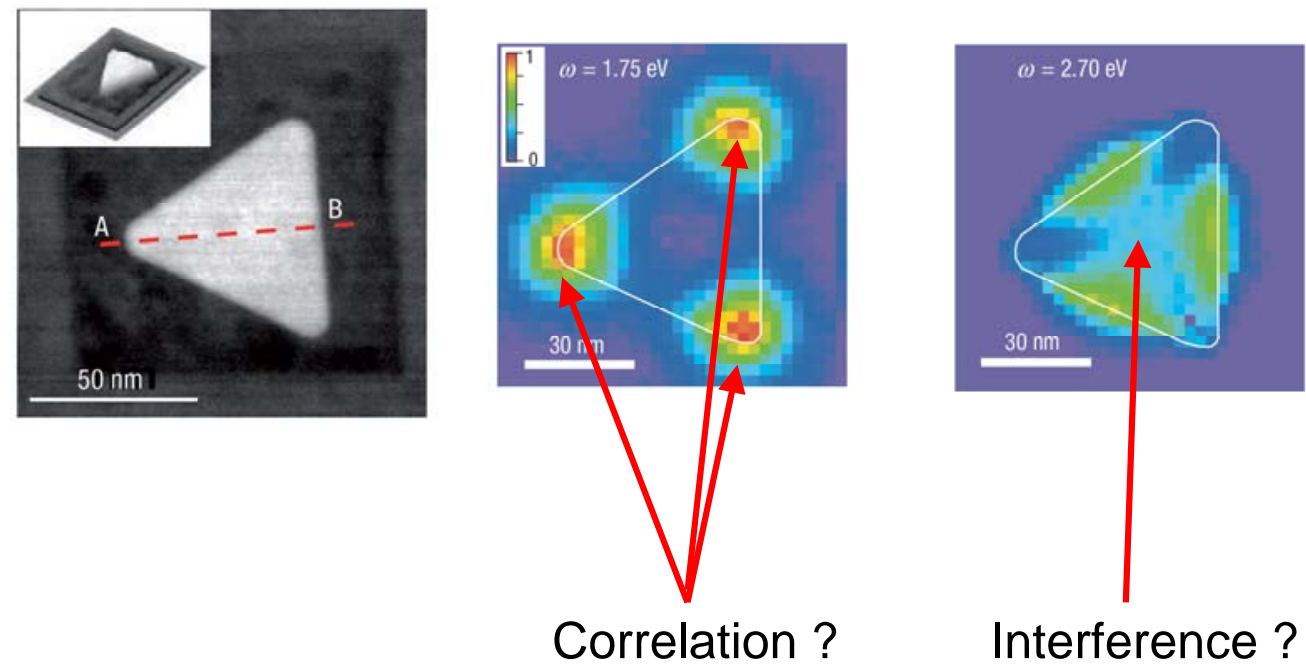

Fig.1 Surface plasmons at a triangular Ag-nanoprism.

The EFTEM-maps recorded for different energies show eigenmode structures in the distribution of excitation of surface-plasmons. These structures are a property of the object. Do the exciting electrons see the whole object and correlation between the excitation peaks? Would they show coherence over the whole particle hence interference phenomena? Example taken from [1].

\section{Electron Interference}

At least in the realm of elastic interaction, TEM is wave optics where the image wave is built up by interference of the waves diffracted at the object. This is a highly complicate situation of multi-beam interference. Much easier to understand is two-beam interference:

Assume a point source emitting an electron. A well-defined spherical wave is propagating in space (fig.2). By means of a wave front splitter, one can select two partial waves. At some distance to the source, these are nearly plane waves described by

$$
\psi_{1 / 2}(x, z ; t)=a_{1,2} \exp ( \pm i \pi k \beta x) \exp (i 2 \pi k z) \exp (i 2 \pi \nu t)
$$

They overlap under a small angle $\beta$; they oscillate at a frequency $v=E$ / $h$ given by the total energy $E$ and Planck's constant $h$. 


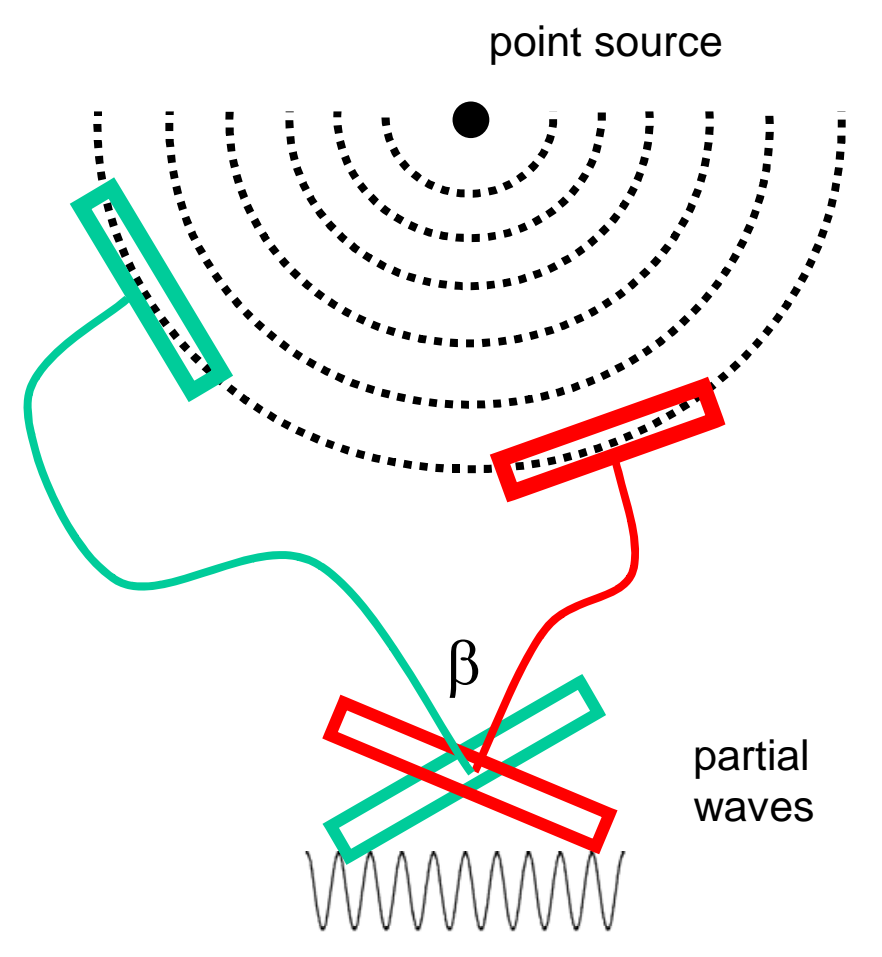

Fig.2 Scheme of Electron Interference

The wave of an electron propagates in space. By some optical arrangement employing beam-splitters and lenses, two partial waves are selected and superimposed in the far field of the source. Since the two partial waves have been part of the same wave, they are mutually coherent hence form an interference pattern. Strictly speaking, this scheme represents only one electron.

In the area of superposition, they give rise to the sum wave

$$
\psi(x, z ; t)=\psi_{1}(x, z ; t)+\psi_{2}(x, z ; t)
$$

with the intensity distribution

$$
I_{h o l}(x)=\psi(x, z ; t) \psi^{*}(x, z ; t)=a_{1}^{2}+a_{2}^{2}+2 a_{1} a_{2} \cos \left(2 \pi q_{c} x\right)
$$

It is a cosinoidal two-beam interference pattern with a "carrier spatial frequency" $q_{c}=k \beta$. This pattern is stationary in time, because both waves oscillate at the same frequency $v$. Please note that the pattern is also independent from $z$, since for simplicity we assumed infinitely extended plane waves.

Experimentally, two beam interferences can most favorably be produced by means of the Möllenstedt electron biprism as a beam splitter (fig.3) [2]. The biprism is a wavefront-splitter, splitting the incoming wave in two partial waves 
and superimposing them downstream by virtue of the attractive electrical field around the positively charged filament.

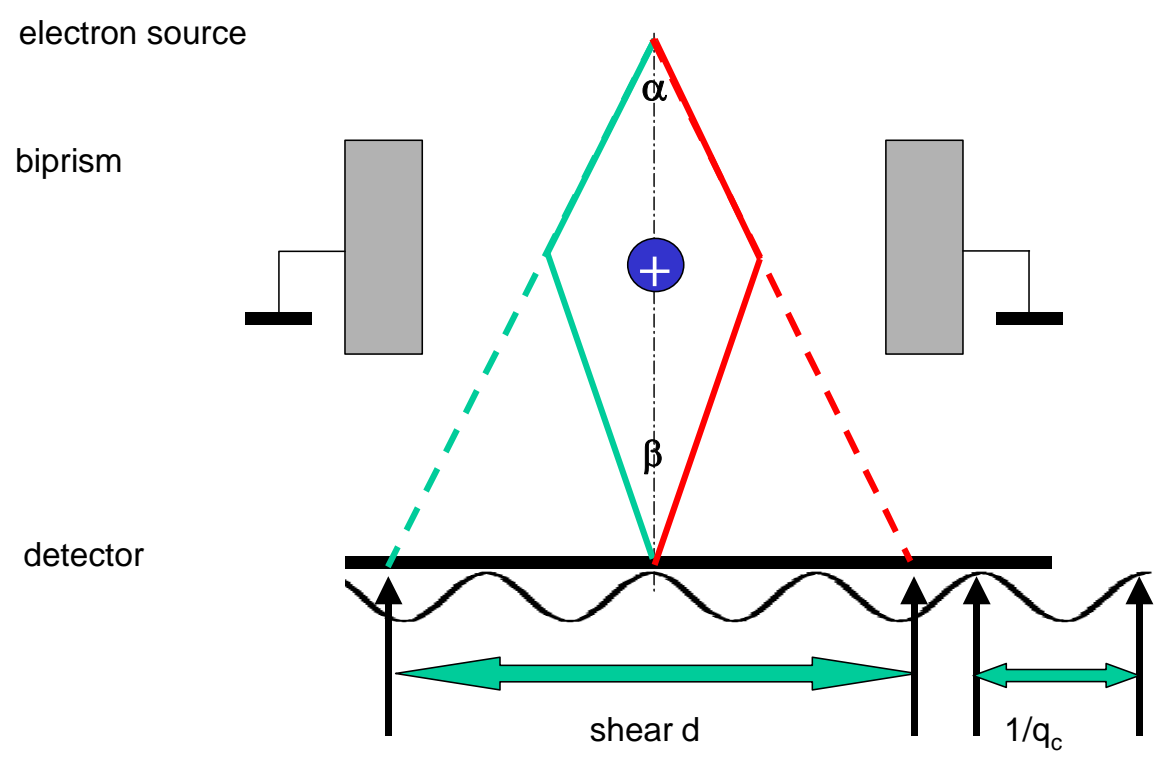

Fig. 3 Setup for two-beam interference using the Möllenstedt electron biprism. By means of the positively charged filament ( $\varnothing \approx 1 \mu \mathrm{m}$, filament voltage several Volt), the incoming wave is split in two partial waves, which are deflected hence superimposed downstream. At biprism voltage $U_{f}=0$, they would be separated in the detector plane by the shear $d$ given by the angular separation $\alpha$ of the two partial waves.

The interference pattern represents the wave of a single electron; it is the probability of finding the electron at the point $(x)$ in the detector plane. In fact, the electron hits the detector somewhere in a very sharp impact (fig.4): The electron is not smeared out over the whole wave area; instead it behaves like a particle, detectable in an a-posteriori well defined but unpredictable position. The unpredictability makes the difference to a classical particle.

Consequently, performing the experiment with a single electron, one cannot see the interference pattern. To see the pattern, one would have to repeat the experiment over and over again with exactly the same electron in the very same experimental setup, in order to fill the probability distribution $I_{h o l}(x)$ with the frequency distribution of many events.

\section{Electron Coherence}

Since, in reality, it is impossible to repeat the experiment with the same electron, we perform the experiment with many different electrons emitted from the source during exposure time (fig.4). 
Different electrons have no fixed phase relation hence must be considered mutually incoherent. However, the phase differences between the respective partial waves of the different electrons in the detector may be very similar. The electrons emitted from different points of the extended source $i\left(\vec{r}_{s}\right)$ contribute with a slightly shifted interference pattern; electrons with different wave number $k$ will contribute with one of a slightly different spatial frequency $q_{c}=k \beta$. All these specific single-electron interference patterns have to be summed up by intensity, i.e. incoherently. Then the intensity distribution resulting from the whole ensemble of recorded electrons is found as

$$
I_{\text {hol }}(x)=a_{1}^{2}+a_{2}^{2}+2 a_{1} a_{2}|\mu| \cos \left(2 \pi q_{c} x+\rho\right)
$$

This averaged pattern differs from the single-electron pattern in the degree of coherence $\mu=|\mu| \exp (i \rho)$, which is a statistical property of the ensemble of all electrons collected on the detector (fig. 5).

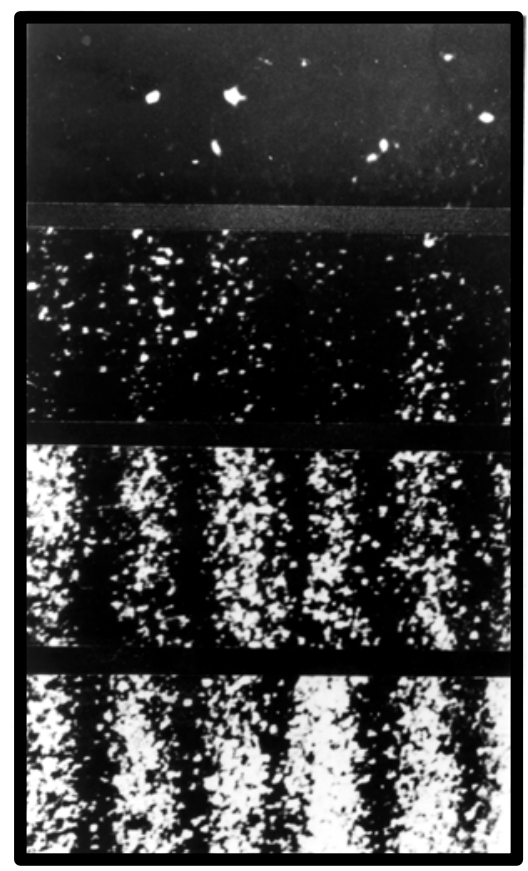

exposure time

$0.02 \mathrm{~s}$

$10 \mathrm{~s}$

$60 \mathrm{~s}$

$120 \mathrm{~s}$

Fig. 4 Built-up of the interference pattern with increasing exposure time.

The electrons drop in one by one at well localizable but unpredictable spots. They are not smeared out over the whole interference pattern. Collecting many electrons at coherent illumination ("ensemble coherence"), the pattern is filled with events and becomes gradually visible. These electrons are mutually incoherent and independent: The time of flight through the microscope is much shorter than $1 \mu \mathrm{s}$, the time distance between two events is about $1 \mathrm{~ms}$; consequently, each electron is virtually all alone in the column ("single electron interference"). If ensemble coherence is sufficient, their individual interference patterns are so similar that by integration an average interference contrast is visible. 


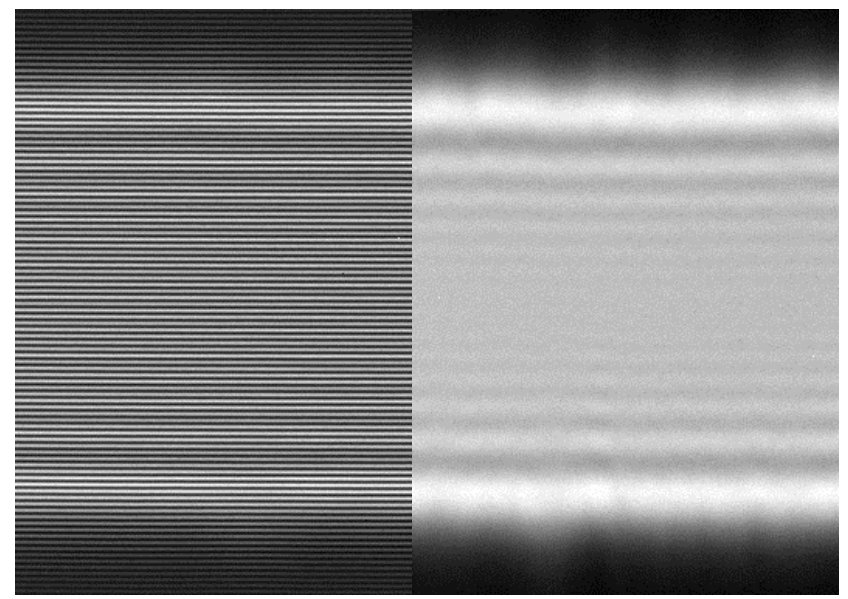

\section{coherent incoherent}

Fig.5 Experimental superposition of two electron waves by means of the electron biprism for demonstration of coherence.

In the left, the two beams are mutually coherent hence form the fine cosinoidal interference fringes. In the right, the interference fringes are missing because of lack of ensemble coherence between the electrons of the two beams; the Fresnel fringes from diffraction of each beam at the respective biprism edge show, however, that there is a certain degree of coherence within each beam. Coherence always describes a bilateral relation: " $a$ " is coherent with " $b$ ".

Therefore we call it "ensemble coherence". It does not say anything about the single-electron wave, which, by definition, is everywhere perfectly coherent with itself, as long as we do not consider inelastic processes.

Under the reasonable assumption that all source points emit the same spectrum of wave numbers, the degree of coherence can be written as

$$
\mu=\mu^{s c} \mu^{t c}
$$

i.e. as the product of spatial coherence $\mu^{s c}$ and temporal coherence $\mu^{\text {tc }}$. Spatial coherence is given as

$$
\mu^{s C}(\vec{\alpha})=\int_{\text {source }} i\left(\vec{r}_{S}\right) \exp \left(i 2 \pi \vec{q} \vec{r}_{S}\right) d \vec{r}_{S}
$$


with $\vec{q}=\left(k_{x}, k_{y}\right)$ and $\vec{\alpha}=\vec{q} / k$ the angular distance at the source of the two partial waves superimposed. ("Van Cittert-Zernike Theorem"). The phase $\rho$ in $\mu=|\mu| \exp (i \rho)$ stems from a possible asymmetry of the source $i\left(r_{s}\right)$.

Temporal coherence may be described by

$$
\mu^{t c}(n)=\int_{\text {source }} s(\kappa) \exp \left(i 2 \pi \kappa n / k_{0}\right) d \kappa
$$

as a function of the order $n$ of interference. It results from the spectrum $s(\kappa)$ with $\kappa=k-k_{0}$ the spectrum coordinate relative to the nominal wave number $k_{0}=\sqrt{2 e m_{0} U_{a}^{*}} / h$, with the assumption $\kappa<<k_{0}$ usually met by far.

Of course, this description of coherence is an approximation. In a more complex situation including dispersion with respect to the incident angle and the energy, e.g. at interaction with the specimen or with aberrations of lenses, one has to determine all the single intensities of the respective wave functions and sum them up incoherently in the final image plane. More details can be found, for example in [3].

\section{Elastic interaction: Phase shift of the wave}

Elastic interaction means that each electron before and behind the object has on the scale of $10^{-15} \mathrm{eV}$ - the same total energy $E$ in the Schrödinger equation. This means that only the direction of the wave vector $\vec{k}$ changes under interaction with the object, but not its modulus $|\vec{k}|$. The coherence properties of the wave are preserved. In the semi-classical WKB-approximation, the wave propagating through an object represented by an electric potential distribution $V$ and a magnetic vector potential $\vec{A}$ is modulated in phase by the so-called eikonal

$$
\varphi=2 \pi \int_{\text {path }} \vec{k} d \vec{s}
$$

which - with respect to a wave propagating in field-free space - results in

$$
\varphi=2 \pi \frac{e}{h v} \int V d s-2 \pi \frac{e}{h} \oint \vec{A} d \vec{s}
$$

where $v$ means the electron velocity; again, details are found in [3]. 


\section{Consequences of energy transfer}

Assume that, for example in the setup described in the following, one of the two coherent partial waves experiences an energy transfer $\delta E$. Then we superimpose

$$
\psi_{2}(x, z ; t)=a_{2} \exp (-i \pi k \beta x) \exp (i 2 \pi k z) \exp (i 2 \pi(v+\delta v) t)
$$

and the unchanged partial wave

$$
\psi_{1}(x, z ; t)=a_{1} \exp (+i \pi k \beta x) \exp (i 2 \pi k z) \exp (i 2 \pi v t)
$$

We assume that in this case entanglement with the object can be neglected; this is justified for the experimental setup used for verification in the following, because any influence from the beam electron on the macroscopic apparatus, such as changing its potential by electrostatic induction, can safely be neglected. Consequently, assuming full ensemble coherence, we find for the resulting interference pattern

$$
I_{h o l}(x, y ; t)=a_{1}^{2}+a_{2}^{2}+2 a_{1} a_{2} \cos \left(2 \pi q_{c} x+2 \pi \delta v t\right)
$$

which is no more stationary in time. Instead, the time dependent phase shift $\varphi(t)=2 \pi \delta v t$ provokes a lateral motion ("beat") of the interference fringes. With $\omega=\partial \varphi / \partial t$, the time dependent phase shift $\varphi(t)$ is associated with an energy transfer $\delta E=\hbar \omega$, according to the Einstein relation. The fringes move by 1 fringe/s, i.e. $\delta v=1 \mathrm{~Hz}$, for $\delta E=4.13510^{-15} \mathrm{eV}$. The effects of the corresponding change of the wave number $k$ with $\delta E$, and hence of the spatial frequency $q_{c}$, are negligibly small.

\section{Experimental Verification}

The time dependent phase shift $\varphi(t)$ was experimentally proven by means of the setup shown in fig.6 [4]. One wave is propagating through field-free space. The other one is passing through a metallic tube, which is connected to a voltage supply providing a voltage linearly increasing in time at a constant rate $\dot{V}$. After time of flight $t_{\text {flight }}$ through the tube, each electron has picked up virtually the same energy $\delta E=e \dot{V} t_{f l i g h t}$, which gives rise to the "beat" frequency $\delta v=\frac{e}{h} \dot{V} t$ flight of the whole interference pattern. 


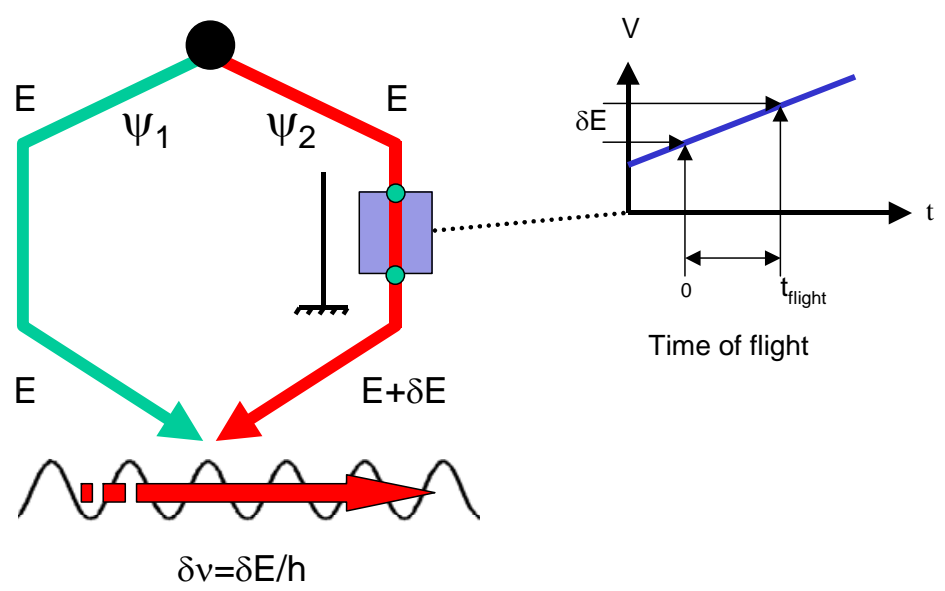

Fig. 6 Beats of electron waves by energy transfer $\delta E$ to one wave.

The wave $\psi_{2}$ is shifted in energy $\delta E$ with respect to the wave $\psi_{1}$. The energy shift comes about under time of flight $t$ flight through the tube with a ramped voltage $V(t)$. Since the fringes move at a frequency $\delta v$, time resolution of the detector decides whether fringe contrast appears (" $\psi_{1}$ and $\psi_{2}$ are found coherent") or not ("incoherent"). From [4].

Interpretation: Loss of coherence between the partial waves It depends on the time resolution $\tau$ of the detector, whether one can still detect interference fringes in the recorded intensity

$$
I_{\text {hol }}(x, y)=2\left(1+\frac{1}{\tau} \int_{0}^{\tau} \cos \left(2 \pi q_{c} x+2 \pi \delta v t\right) d t\right)
$$

which can be written as

$$
I_{\text {hol }}(x, y)=2\left(1+\left|\mu^{\delta E}(\tau)\right| \cos \left(2 \pi q_{c} x+2 \pi \delta v \tau / 2\right)\right)
$$

with

$$
\mu^{\delta E}(\tau)=\left|\mu^{\delta E}(\tau)\right| \exp (i 2 \pi \delta v \tau / 2)=\frac{1}{\tau} \int_{0}^{\tau} \exp (i 2 \pi \delta v) d t
$$

For $\delta v=$ const one obtains

$$
\left|\mu^{\delta E}(\tau)\right|=\frac{\sin (\pi \delta v \tau)}{\pi \delta v \tau}
$$

with the first zero given at $\tau=1 / \delta v$. Consequently, it depends strongly on exposure time $\tau$, whether interference contrast, i.e. coherence, is found or not. The detector is an essential part of the whole experiment. For the usual 
exposure times of about $1 \mathrm{~s}$, the energy threshold for "decoherence" is $\approx 4.13510^{-15} \mathrm{eV}$.

This coherence $\mu^{\delta E}$ is in principle different from "ensemble coherence": The disappearance of the interference contrast would show up also at perfect ensemble coherence. Instead, here we deal with the coherence between the partial waves of the same state, because we encroach on the very state of the single electron by energy transfer with the consequence of destroying coherence with other parts of the previous wave; without energy transfer we would simply produce a phase shift under conservation of coherence. Therefore, we call this kind of coherence "state coherence" $\mu^{\text {state }}$, which equals 1 for elastic interaction. The difference between ensemble coherence and state coherence is the reason, why the comparably large energy spread of the electron source of $\Delta E \approx 1 \mathrm{eV}$ entering temporal ensemble coherence may be many orders of magnitude larger than $4.13510^{-15} \mathrm{eV}$. Altogether, we have now the total degree of coherence

$$
\mu=\mu^{s c} \mu^{t c} \mu^{\text {state }}
$$

In the above example, $\mu^{\text {state }}=\mu^{\delta E}$ holds. In the following case, $\mu^{\text {state }}$ will describe the distribution of coherence in a wave created by inelastic scattering.

\section{Inelastic coherence}

Compared to $4.135 \cdot 10^{-15} \mathrm{eV}$, the energy transfer to a wave usually occurring at inelastic interaction is huge. Therefore, excitation of phonons with several $m e V$, of plasmons at several $\mathrm{eV}$, or of inner shell excitations at several $10 \mathrm{eV}$ necessarily destroys the degree of state coherence with respect to an elastically scattered wave, or to an unscattered one: The beat frequency would be about $10^{12} \div 10^{17} \mathrm{~Hz}$, and, after usual exposure times in the order of seconds, interference contrast in an image-plane hologram would be completely wiped out.

Of course, considering the object simply as a source of inelastic electrons, the issued inelastic waves can be used for producing interference in the far-field of the object according to the scheme in fig.2; then the respective ensemble coherence - now given by the energy distribution delivered by the electron gun convolved with the energy spread from the inelastic events, and the size of the object - determines the interference contrast.

The much more interesting question is, whether there is extended coherence $\mu^{\text {state }}$ within the arisen inelastic wave in the inelastically scattering volume, i.e. in the object or image, which would reveal wave-like phenomena, when adjacent areas are superimposed. In the following, we analyze this in more detail. We 
start with an illuminating wave with infinite region of coherence per definition, i.e. state coherence $\left|\mu^{\text {state }}\right| \equiv 1$. In the scattered wave field of a single electron, the coherence distribution is determined by the nature of the scattering process. For example, purely elastic scattering is modulating the wave field in amplitude and phase without affecting coherence. But in case of an inelastic scattering event, such as a K-shell ionization in one oxygen atom, the electron is exciting the object locally. Thereby, the illuminating, extended wave collapses and a new one is "born" locally. For understanding, we have to investigate, whether or not we find extended coherence $\mu^{\text {state }}$ indicating the extension of the newborn inelastic wave. This is a non-trivial situation, because for coherence we need a fixed phase relation, which is not automatically guaranteed over a larger area. The equality of energy better than $4 \cdot 135 \cdot 10^{-15} \mathrm{eV}$ is a necessary condition, but, presumably, not a sufficient one.

For investigation of the coherence properties of the inelastic electrons, we record holographic EFTEM-images by means of the setup shown in fig.7. Interferograms are recorded in the image plane of the object such that two closely adjacent areas of the inelastic field overlap. First findings with Alplasmons revealed coherence [5], and, with improved instrumentation, the extension of coherence was estimated $>10 \mathrm{~nm}$ [6]. Finally, the systematic investigation increasing the shear $d$ (fig.8) resulted in the coherence distribution shown in fig.9-10 [7]. Please note that these measurements were performed in the image plane of the object; this means that we measure the coherent extension of the scattered wave at the scatterer. 


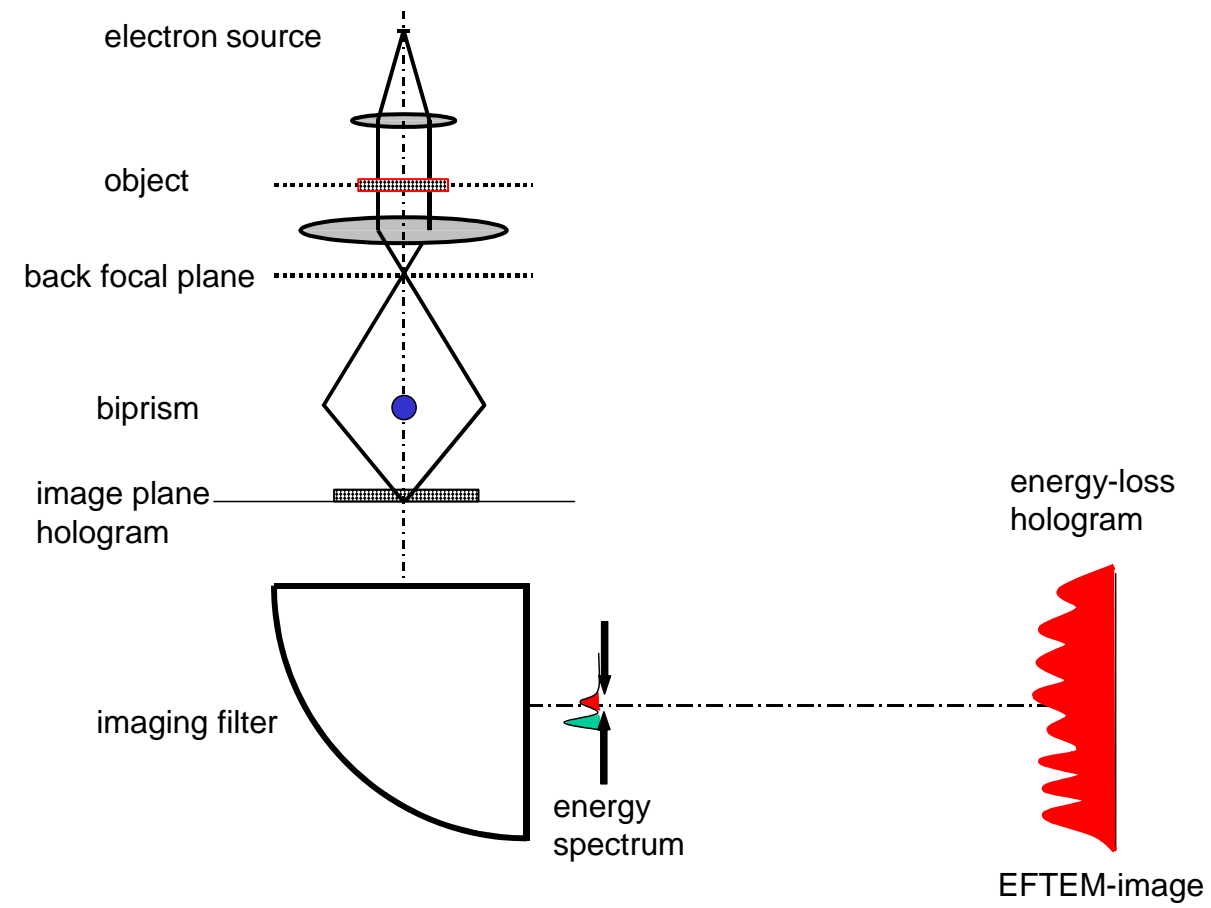

Fig. 7 Setup for recording holograms with inelastically scattered electrons in an EFTEM. Both beams subsequently superimposed go through the object to make sure that both may suffer the same energy transfer at e.g. plasmon scattering. From the hologram built up by all electrons, elastic and inelastic, we select a certain window in the energy spectrum to reveal the corresponding energy-loss hologram. Please keep in mind that this is EFTEM-imaging, meaning that we have an image-plane hologram of the inelastic process. 


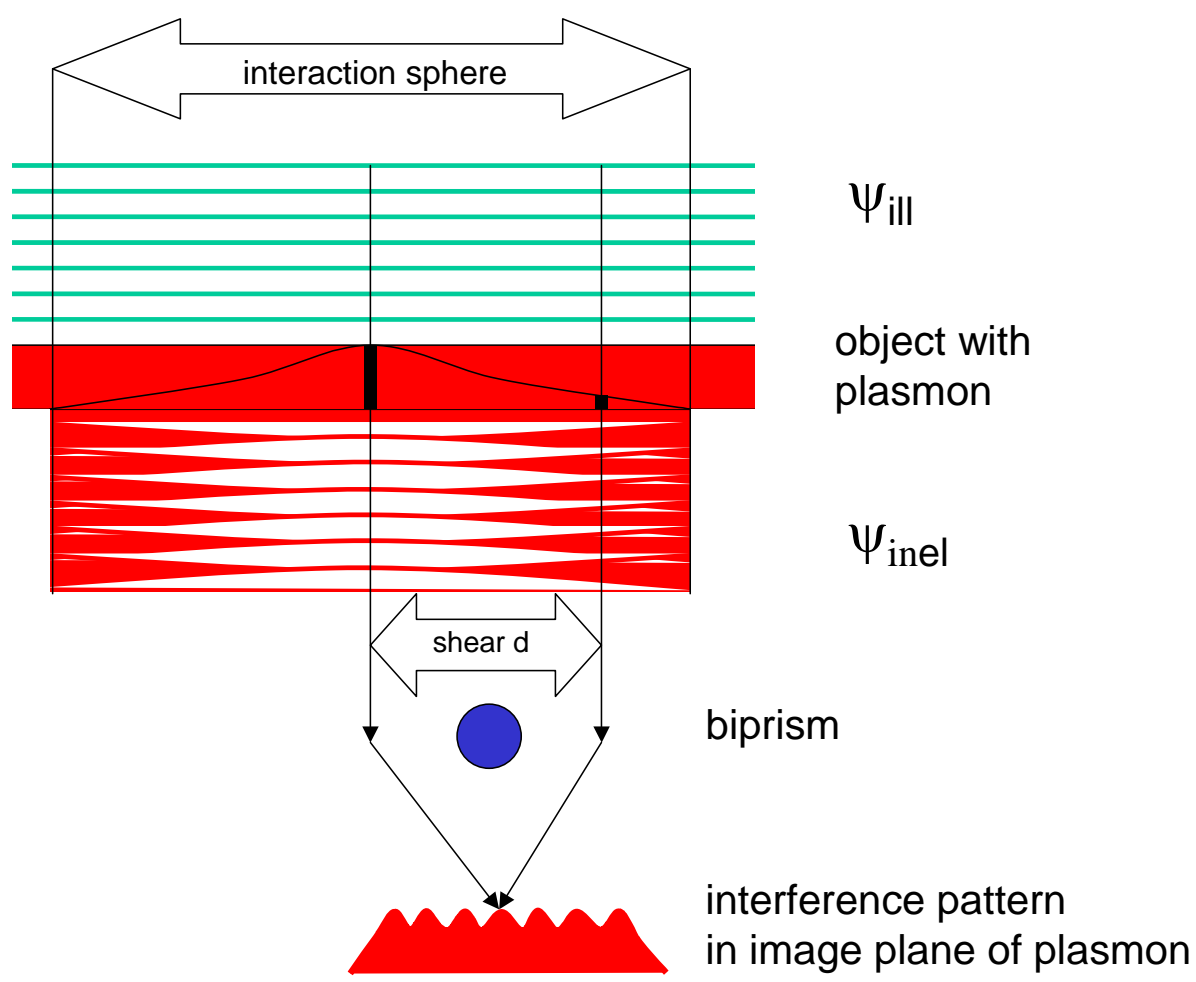

Fig. 8 Scheme for measuring the extension of coherence in inelastic scattering.

The plasmon is the source of the inelastic wave. We measure the extension of this source by measuring the coherent extension of the issued inelastic wave in the plasmon plane as follows: By means of the biprism voltage, the shear $d$ is increased. The contrasts of the respective hologram-fringes give the distribution of degree of coherence $\mu^{\text {state }}$ with $d$. The optics for imaging the plasmon into the interference plane is not drawn. 
Zero
loss
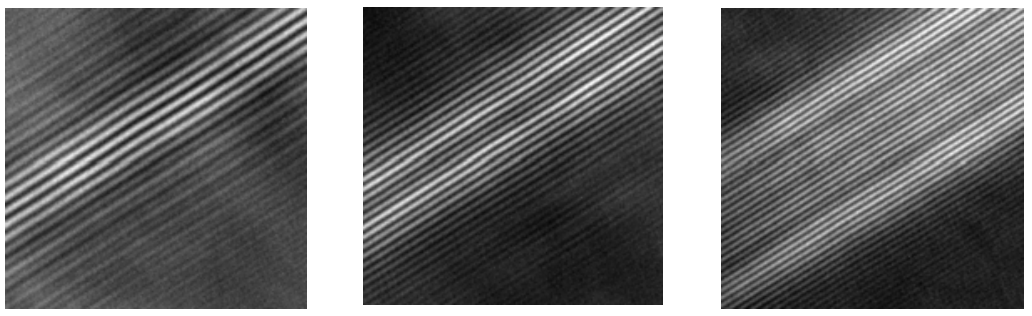

\section{Plasmon loss}

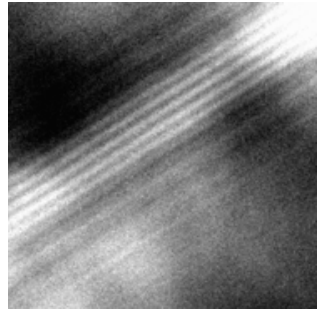

$13.2 \mathrm{~nm}$

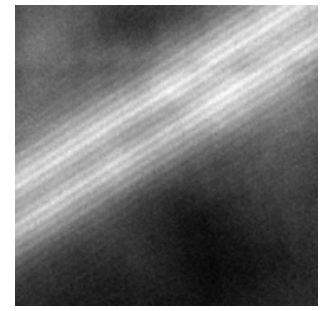

19.6nm

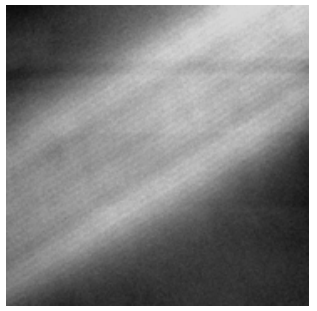

$24.7 \mathrm{~nm}$

Fig. 9 Electron holograms recorded with plasmon-scattered electrons at increasing shear. With increasing shear, fringe spacing decreases and overlapping width increases. For subsequent normalization with the "ensemble coherence" of illumination, also the zero-loss holograms are recorded. The result is $\mu^{\text {state }}$ in the plasmon-scattered wave. Expectedly, the fringe contrasts in the plasmon-loss holograms are much lower than in the respective zero-loss holograms. For details see [7].

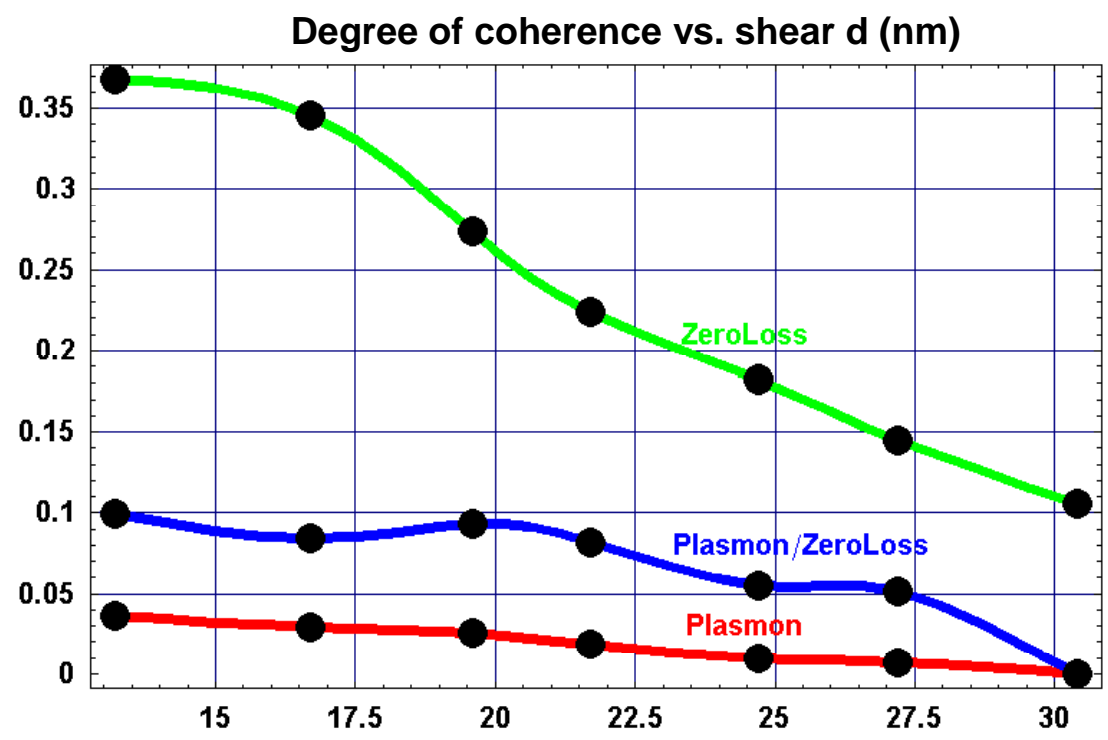

Fig. 10 Extension of coherence within in the inelastically scattered wave field.

The curve Plasmon/Zeroloss gives $\mu^{\text {state }}$ of the plasmon scattered electrons. Surprisingly, in spite of the generally poor coherence, coherent contributions have to be considered in areas up to 30nm wide at plasmon scattering. This is interpreted as the diameter of the newborn inelastic wave at the scatterer. From data of fig. 8. 
The interpretation is that there is an outgoing inelastic wave, which, contrary to elastic scattering, is now spatially restricted to a so-called interaction sphere of a certain inelastic scattering event (fig.11). This sphere is the dominating factor contributing to the region of coherence in the inelastically scattered wave field, as shown in $[10,11]$. Furthermore it was shown that the shape of the coherence region is given by the delocalized Coulomb field of the scattered electron and also determined by the physical properties of the object such as correlations in quasi-particle excitations, like plasmons, polaritons or phonons. The radius of the interaction sphere is determined by the amount of transferred energy and beam energy of the incident electron [8].

elastic

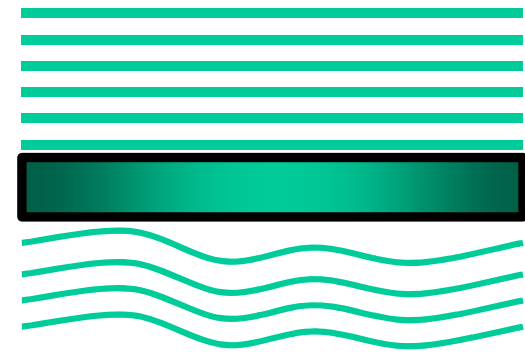

$\psi_{\mathrm{el}}$ inelastic

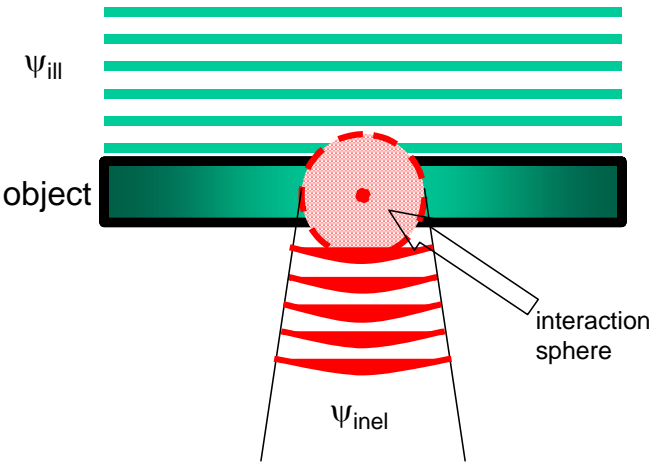

Fig.11 Elastic and inelastic interaction of the electron wave with an object Left: At elastic interaction, the object exit wave $\psi_{e l}$ is phase-modulated according to the electric and magnetic object potential under preservation of coherence properties. Right: At inelastic interaction, the illuminating wave $\psi_{\text {ill }}$ collapses and gives rise to a "newborn" inelastic wave $\psi_{\text {inel }}$ in the interaction sphere shown; the extension of the inelastic wave is determined by the coherence measurements. At the edge of an object, it may also reach out laterally into vacuum [7].

\section{Density Matrix description}

As a useful construction for further discussion, we use the more general density matrix approach, which is fully equivalent to the above wave function description. In case of pure states, the density matrix of a fast electron can be set up by multiplying the wave with its complex conjugate for all pairs of independent variables $\left(x, x^{\prime}\right)[9]$ :

$$
\rho\left(x, x^{\prime}\right)=\psi(x) \psi^{*}\left(x^{\prime}\right)
$$

$x-x^{\prime}$ is the shear $d$ introduced above. 
In case of real electron sources we have to regard an ensemble of beam electrons, which are mutually incoherent, generally described by a density matrix of a mixed state.

$$
\rho\left(x, x^{\prime}\right)=\int_{\text {Soucre }} f(\vec{k}) \psi_{\vec{k}}(x) \psi_{\vec{k}}^{*}\left(x^{\prime}\right) d^{3} k
$$

The integration runs over the energy and angular distribution in the electron beam. The distribution function has to be normalized

$$
\int_{\text {Source }} f(\vec{k}) d^{3} k=1
$$

The emitted electron waves, which differ in energy and incident direction, can be written with respect to object plane as

$$
\psi_{\vec{k}}(x)=\psi_{0}(x) \exp \left(2 \pi i k_{\perp} x\right)
$$

This ansatz leads to simplification of the mixed state density matrix of the beam electrons:

$$
\begin{aligned}
\rho\left(x, x^{\prime}\right) & =\psi_{0}(x) \psi_{0}^{*}\left(x^{\prime}\right) \int_{\text {Source }} f(\vec{k}) \exp \left(2 \pi i\left(x-x^{\prime}\right)\right) d^{3} k \\
& =\psi_{0}(x) \psi_{0}^{*}\left(x^{\prime}\right) \mu\left(x-x^{\prime}\right) \\
& =\rho_{\text {in }}\left(x, x^{\prime}\right) \mu\left(x-x^{\prime}\right)
\end{aligned}
$$

It collapses to a product of the incoming density matrix $\rho_{\text {in }}\left(x, x^{\prime}\right)$ and the complex degree of coherence $\mu\left(x-x^{\prime}\right)$. It shows directly that the off-diagonal elements are weighted by a factor, which is decreasing with increasing relative distance $x-x^{\prime}$ for standard electron sources.

For simplicity we assume to have an ideal, non-dispersing lens for the further imaging process. This is justified if we investigate waves, which are slowly varying over a length scale of $10 \mathrm{~nm}$. This assumption allows that we only need to alter the incoming density matrix by the scattering process by the object, but do not need to average over the coherent wave transfer function of the optics for different incident beams.

The scattering by the object leads generally to an entanglement with (orthogonal) object states. The measurement of beam electrons is described by integration over all degrees of freedom of the object ending up in reduced density matrix $\rho_{\text {out }}\left(x, x^{\prime}\right)$ [9]. 


$$
\rho_{\text {in }}\left(x, x^{\prime}\right) \rightarrow \rho_{\text {out }}\left(x, x^{\prime}\right)=\sum_{i=1} \psi_{i}(x) \psi_{i}^{*}\left(x^{\prime}\right)
$$

We will see that the coherence of the whole beam ensemble is represented by non-diagonal elements of the reduced density matrix of the inelastically scattered fast electron. However, the diagonal elements are the only measurable parts of the density matrix. They represent the intensity distribution in a hologram and in a conventional TEM image [10]

$$
I(x)=\left.\rho_{\text {out }}\left(x, x^{\prime}\right)\right|_{x=x^{\prime}}=\sum_{i=1}\left|\psi_{i}(x)\right|^{2}
$$

This corresponds to an incoherent superposition of different inelastically scattered electrons as usually treated in scattering theory. Fortunately, by means of a biprism it is possible to shift off-diagonal elements into the diagonal, which makes them measurable by means of Off-Axis Electron Holography [11]. In terms of the density matrix approach, the hologram intensity for a certain shear $d$ may be written as follows [10]:

$$
I_{\text {hol }}(x)=\rho_{\text {hol }}(x, x)=\rho_{\text {out }}(x+d / 2, x+d / 2)+\rho_{\text {out }}(x-d / 2, x-d / 2)+2 \Re[\underbrace{\left[\mu(d) \rho_{\text {out }}(x-d / 2, x+d / 2)\right.}_{\text {off }- \text { diagonal element }}]
$$

This expression compared to equation (4) shows that the off-diagonal elements determine the interference term hence also the coherence. This coherence corresponds to ensemble coherence of the beam electrons $\mu(d)$ and to the coherence due to all possible inelastic scattering processes $\rho_{\text {out }}(x-d / 2, x+d / 2)$ for certain shear $d$. The ensemble coherence of the incident fast electrons can be determined directly in a reference measurement without object under the same illumination parameters for the imaging conditions as assumed above.

The reduced density matrix for the fast electron after an inelastic scattering event with energy transfer $\delta E$ can be written as [14]

$$
\rho_{\text {out }}\left(x, x^{\prime}\right)=\left(\frac{2 \pi m e^{2}}{\varepsilon_{0} \hbar^{2} k}\right)^{2} K_{0}\left(q_{E}|x|\right) K_{0}\left(q_{E} \mid x^{\prime}\right) \otimes S\left(x, x^{\prime}, \delta E\right)
$$

with $\otimes$ meaning convolution.

This expression combines the influence of the Coulomb delocalization of the inelastic interaction with the correlations in the excited quasi-particle field. The interaction sphere is described by the Bessel functions $K_{0}$ of second kind and zeroth order, parameterized by the characteristic scattering vector 
$q_{E}=\delta E /(2 E) k_{0}$. The correlation in the quasi-particle excitation is denoted by the density-density correlation function [14]

$$
S\left(x, x^{\prime}, \delta E\right)=\int_{-\infty}^{+\infty} \int_{-\delta E}^{0} \rho_{i}\left(\vec{r}, \vec{r}^{\prime}, \varepsilon\right) \rho_{f}\left(\vec{r}^{\prime}, \vec{r}, \varepsilon+\delta E\right) d \varepsilon \exp \left(-i q_{E}\left(z-z^{\prime}\right)\right) d z d z^{\prime}
$$

with $\vec{r}=(x, z), \rho_{i}$ and $\rho_{f}$ the density matrices of initial and final state of the object with energy difference $\delta E$, and $\varepsilon$ the integration variable in energy, respectively; it is the 2D-Fourier transform of the mixed dynamic form factor (MDFF). In case of dipole transitions, some reasonable approximations confirm the experimental results for bulk plasmons satisfactorily [10-13]. In these cases, $S\left(x, x^{\prime}, \delta E\right)$ is strongly attenuated for length scales larger than $1 \mathrm{~nm}$, whereas the whole interaction sphere extends to about $10 \mathrm{~nm}$ for excitations with $15 \mathrm{eV}$ energy transfer. This means that the holographically measured coherence in the outgoing wave field is here determined rather by the Coulomb delocalization than by the correlation within the collective excitation [10]. Since, at the end, we are interested in correlations within quasi-particle excitations, we need to either reduce the Coulomb delocalization by decreasing beam energy or look for excitations, which provide a correlation length comparable to the interaction sphere extension. We will exhibit that surface plasmons might be promising candidates for those investigations.

\section{Experiments with surface plasmons}

During the last years, astonishing works were presented concerning the modulation of coherence of light due to surface plasmon scattering [15-17]. They also motivated us for the study of coherence properties of inelastically scattered electrons on plane metal surfaces.

For this purpose, holes with rectangular shape were etched in a thin aluminium foil by means of Focused Ion Beam (FIB). A platinum bar was deposited before etching, to mask a freestanding aluminium bar, which provides geometrically well-defined plane surfaces in beam direction. Since the etching process was not homogeneous over the whole area, the upper half of the Al bar is still covered with platinum, whereas the lower half seems to be pure aluminium with the exception of a thin platinum line at the lower edge (fig.12, left). Also the bright edges correspond to the pure Al bar, which is located underneath the partially with Pt contaminated surface. By means of the Gatan Imaging Filter (GIF 200) at a CM200 FEG Transmission Electron Microscope, Energy Spectroscopic Imaging (ESI) was performed in order to verify the existence of surface plasmons at the edges to the vacuum by means of averaged local spectra (fig. 12, right). For all measurements, the width of the energy-selecting slit was set to 1 $\mathrm{eV}$ and the offset to the high tension was increased in $1 \mathrm{eV}$ steps from -2eV to $18 \mathrm{eV}$. In order to increase the inelastic cross section, the high tension was 
reduced to $80 \mathrm{kV}$. Resonances at about $7 \mathrm{eV}$ and $15 \mathrm{eV}$ were found at the lower edge, whereas at the upper edge only the bulk resonance of the aluminium plasmon at $15 \mathrm{eV}$ [18] appeared (fig. 12, right). We associate the $7 \mathrm{eV}$ resonance to surface plasmons of the aluminium bar in presence of platinum as created by FIB; the values of $6.7 \mathrm{eV}$ for aluminium spheres [19] and $10.5 \mathrm{eV}$ for plane aluminium surfaces [20] indicate a strong shape dependence of the resonance energies. The $7 \mathrm{eV}$ surface resonance found here is of interest for the following coherence measurements. The upper edge will serve as a reference region for a measurement without that surface plasmon, which might be suppressed through the presence of the platinum coverage.
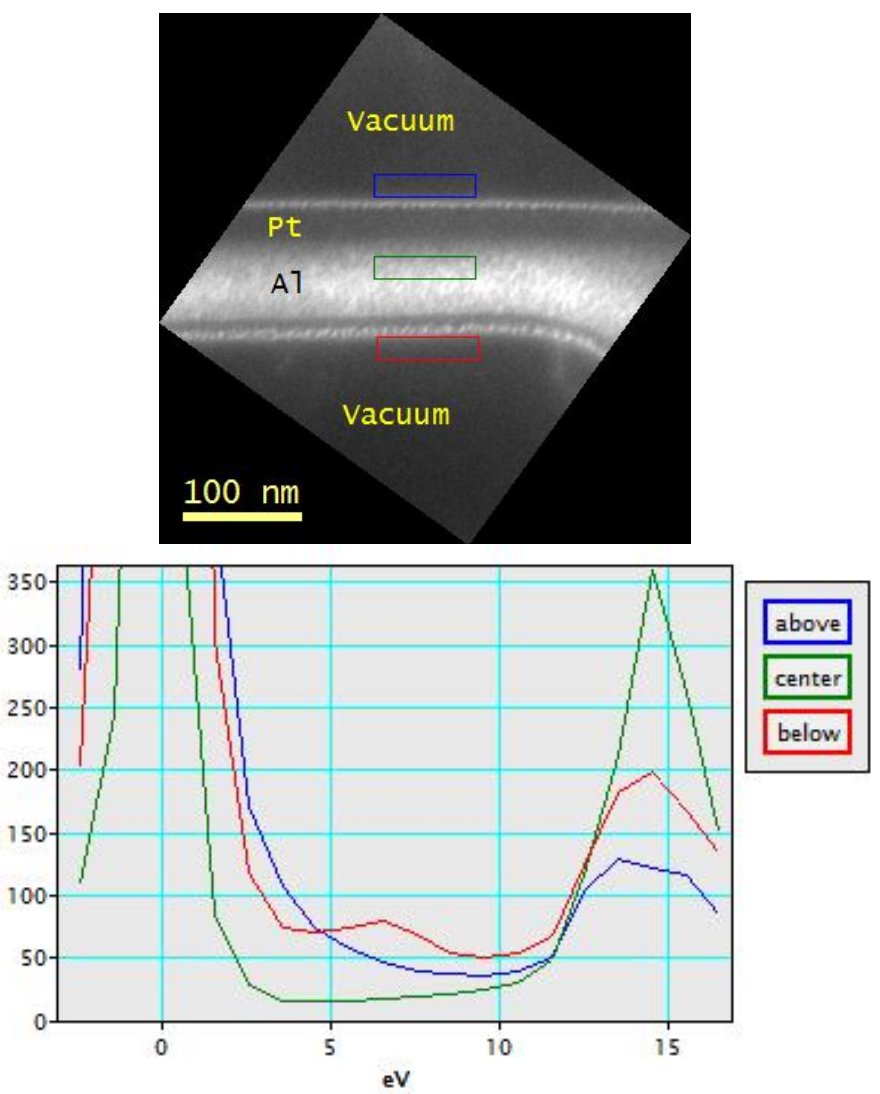

Fig. 12 Aluminium plasmon energy-loss image (15eV energy loss, leV slit-width) of aluminium bar with averaged spectra at three different positions: red: below, green: in the middle of, blue: above the bar. The filtered image shows a dark area in the upper part of the bar due to platinum remaining from FIB preparation (Platinum plasmon loss: $35 \mathrm{eV}$ [17]). The bright band shows the more or less pure aluminium part. The spectra reveal a surface plasmon resonance at about $7 e \mathrm{~V}$ below the edge (red), whereas a broader tail without resonance is indicated by the spectrum above the bar (blue).

Interference fringes were generated by means of a biprism oriented perpendicularly to the edge of the above-mentioned aluminium bar. Since the infocus image appears slightly above the biprism plane, a negative voltage has to be applied in order to generate interference fringes within the first image 
plane. Fringes above and below the edge were recorded in an energy-filtered series with the mentioned filter settings. The results are obtained by evaluating the contrast in each line parallel to the edge, which is shown in figs. $13 a$ and $b$ for $7 \mathrm{eV}$ filtered electron holograms. The modulus of the degree of coherence given by the fringe contrast

$$
|\mu|=\frac{I_{\max }-I_{\min }}{I_{\max }+I_{\min }}
$$

is measured by the ratio of difference and sum of maximum and minimum local intensity:
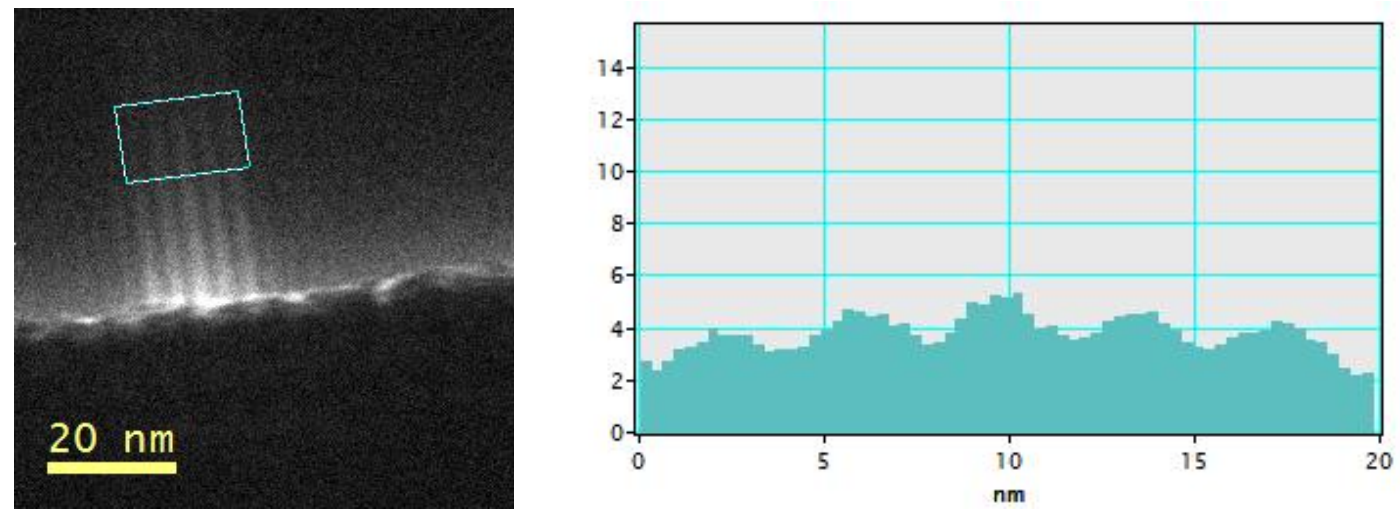

Fig. 13a Energy-loss filtered electron hologram ( $7 e V$ ) of vacuum region and corresponding contrast profile $25 \mathrm{~nm}$ above the edge of the bar. The averaged and zeroloss normalized (i.e. corrected for ensemble coherence) contrast for this profile is about 0.35 .
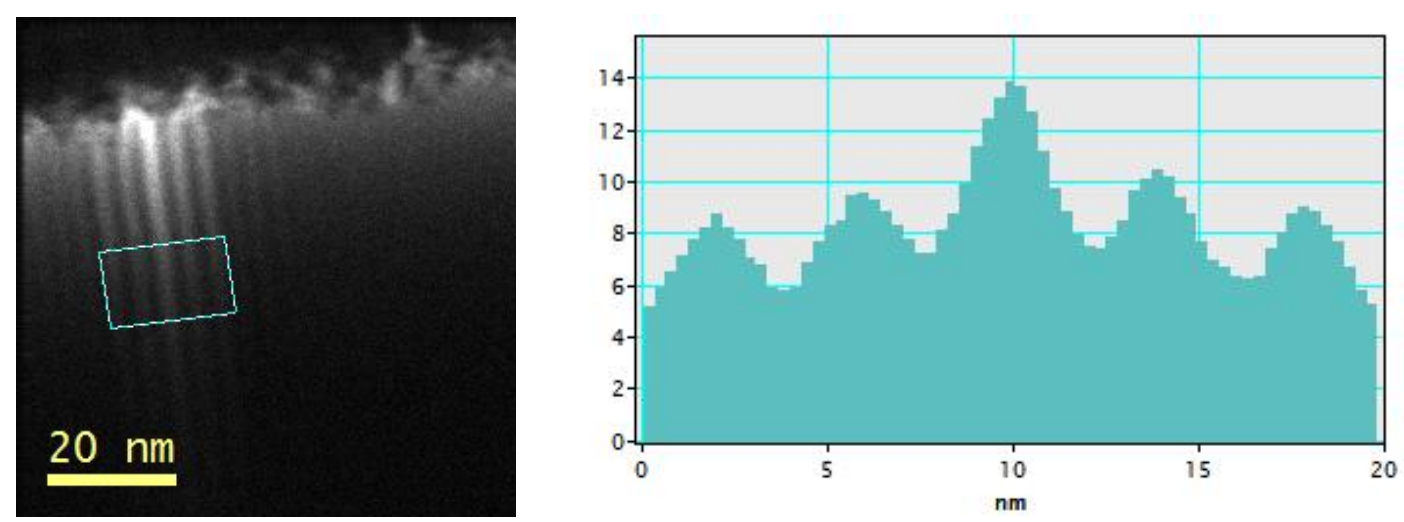

Fig. 13b Energy-loss filtered electron hologram ( $7 \mathrm{eV}$ ) of vacuum region and corresponding contrast profile $25 \mathrm{~nm}$ below the edge of the bar. The averaged and zeroloss normalized (i.e. corrected for ensemble coherence) contrast for this profile is about 0.50 . 
For evaluation of contrast we chose vacuum regions at a distance of $25 \mathrm{~nm}$ to the edges. A difference of about 0.15 in mean contrast is observable between fringe profiles above and below the edge. To enhance the significance, the acquisition of the filtered series was repeated 5 times for the reference area above bar and 8 times for the surface plasmon resonance area below bar. The subsequently averaged contrast values appear with reduced noise. A further statistical evaluation gives access to rough estimates for error bars.

\section{Results}

The evaluation of the fringe contrast in both vacuum regions leads to significant differences for the regions above and below the aluminium bar. The measured contrast was normalized by the contrast of the zero-loss hologram fringes, in order to eliminate the contrast attenuation by ensemble coherence within the electron beam (Eqn. 4). The determination of fringe contrast is performed for each image of the filtered series, which provides a function of contrast in dependence on energy loss. Such a series was already examined in [13] by an energy filtered hologram series for bulk aluminium, but without surface resonances. The normalized contrast is represented in dependence on energy.

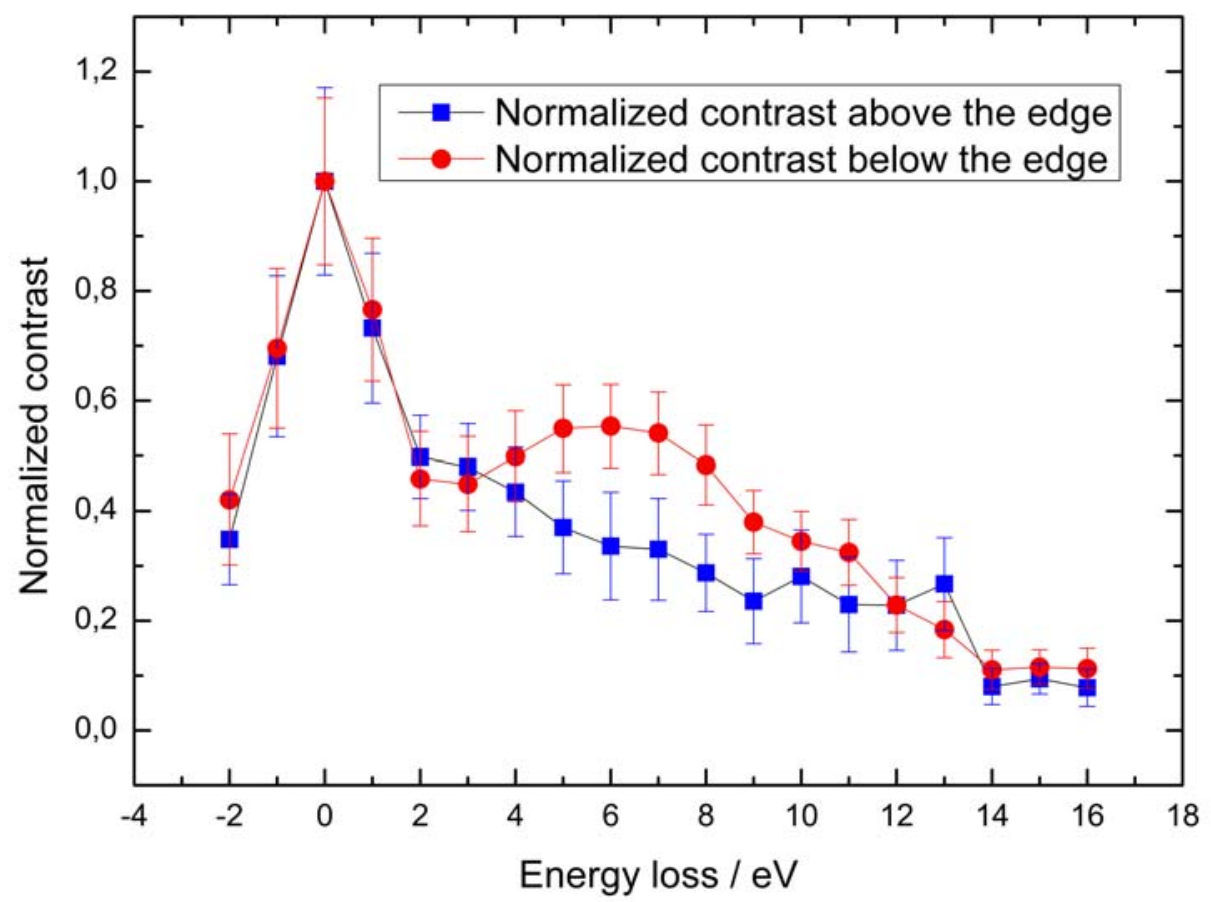

Fig. 14 Normalized mean contrast of the fringes $25 \mathrm{~nm}$ above (blue) and below (red) the respective edge. The red curve shows a maximum around beV, whereas the blue curve decreases monotonically. The contrast maximum is attributed to the surface plasmons. 
The contrast is changing also in dependence on the distance from the edge. It appears that the contrast corresponding to the resonance increases with increasing distance to the edge, which is a similar behaviour as predicted in [10] and already measured in [7]. A very interesting explanation for that in a way counterintuitive result can be found in [21] in terms of an interpretation of the famous double slit experiment.

These very first results show that a significant increase of contrast for the fringe pattern below the edge in the region between 5-7eV (fig. 14, red) coincides with the presence of a surface plasmon resonance in the spectrum of fig. 12 (right); on the contrary, the reference area above the edge only shows a monotonic decrease of contrast with increasing energy loss. Further experiments are necessary to increase the signal to noise ratio in the extracted contrast plots in order to interpret all details of the contrast profiles.

\section{Conclusions}

We interpret our findings as a correlation between the nature of the surface plasmons with the increase of coherence in the surface plasmon scattered wave field. The findings cannot be explained by a simple increase of intensity due to a higher excitation probability from the surface plasmon, because this increase would be proportional to $I_{\max }$ and $I_{\min }$ in the same way, hence would cancel out directly in the evaluating contrast formula

$$
|\mu|=\frac{I_{\max }-I_{\min }}{I_{\max }+I_{\min }}
$$

Consequently, because the Coulomb delocalization function $K_{0}$ is the same for both measurements above and below the bar (at least in the vacuum region), the increase of coherence must be related to object properties. This means that the density-density correlation function $S\left(x, x^{\prime}, \delta E\right)$ for a surface plasmon appears to be the dominating factor. It is different in bulk plasmon scattered wave fields, where extension of coherence is predominantly determined by Coulomb delocalization [10].

The results are encouraging for further studies of coherence effects in inelastically scattered wave field. These studies are necessary to obtain a deeper insight into the nature of collective excitations in solids, which become more and more important in technologic applications, e.g. in the field of plasmonics. The possibilities of inelastic holography for investigating surface plasmon coherence will be outstandingly interesting for understanding the very beautiful surface plasmon eigen-modes in triangular silver nano-particles by answering the initial question: Do the electrons see the whole shape of a nanoparticle? 


\section{References}

[1] J. Nelayah, M. Kociak, O. Stephan, F.J. Garcia de Abajo, M. Tence, L. Henrard, D. Taverna, I. Pastoriza-Santos, L.M. Liz-Marzan and Ch. Colliex: Mapping surface plasmons on a single metallic nanoparticle, Nature Physics 2007, doi:10.1038/nphys575

[2] G. Möllenstedt and H. Düker: Beobachtungen und Messungen an BiprismaInterferenzen mit Elektronenwellen, Z. Physik, Vol. 145 (1956) 377 [3] H. Lichte and M. Lehmann: Electron holography - basics and applications, Rep. Prog. Phys. 71(2008) 016102 (Online at stacks.iop.org/RoPP/71/016102) [4] H. Schmid: Ein Elektronen-Interferometer mit 300 um weit getrennten kohärenten Teilbündeln zur Erzeugung hoher Gangunterschiede und Messung der Phasenschiebung durch das magnetische Vektorpotential bei metallisch abgeschirmtem Magnetfluss. Thesis (1985), University of Tübingen

[5] A. Harscher, H. Lichte and J. Meyer: Interference experiments with energy filtered electrons, Ultramicroscopy 69 (1997) 201-209

[6] H. Lichte and B. Freitag: Inelastic electron holography, Ultramicroscopy 81 (2000) 177-186

[7] P.L. Potapov, H. Lichte, J. Verbeeck and D. van Dyck: Experiments on inelastic electron holography, Ultramicroscopy 106 (2006) 1012-1018

[8] H. Rose: Image formation by inelastically scattered electrons in electron mircoscopy, Optik, 45 (1976) No 2 p. 139-158

[9] M. Schlosshauer: Decoherence and the quantum-to-classical transition; 2007 Springer-Verlag Berlin Heidelberg, Corrected third printing 2008.

[10] P. Schattschneider and H. Lichte: Correlation and the density-matrix approach to inelastic electron holography in solid-state plasmas, Phys. Rev. B 71 (2005) 045130

[11] J. Verbeeck, D. van Dyck, H. Lichte, P. Potapov and P. Schattschneider: Plasmon holographic experiments: theoretical framework, Ultramicroscopy 102 (2005) 239-255

[12] H. Kohl and H. Rose: Theory of Image Formation by Inelastically Scattered Electrons in the Electron Microscope; Advances in Electronics and Electron Physics Vol. 65 (1985) 173-224

[13] J. Verbeeck, G. Bertoni and P. Schattschneider: The Fresnel effect of a defocused biprism on the fringes in inelastic holography, Ultramicroscopy 108 (2008) 263-269

[14] P. Schattschneider, M. Nelhiebel and B. Jouffrey: Density Matrix of inelastically scattered fast electrons, PRB 59 (1998) 16

[15] H.F. Schouten, N. Kuzmin, G. Dubois, T.D. Visser, G. Gbur, P.F.A. Alkemade, H. Blok, G.W.'t Hooft, D. Lenstra and E.R. Eliel: Plasmon-Assisted Two-Slit Transmission: Young's Experiment Revisited, PRL 94, 053901 (2005)

[16] C. H. Gan, G. Gbur and T.D. Visser: Surface Plasmons Modulate the Spatial Coherence of Light in Young's Interference Experiment, PRL 98, 043908 (2007) 
[17] S. Ravets, J.C. Rodier, B. Ea Kim, J.P. Hugonin, L. Jacubowiez and P. Lalanne: Surface plasmons in the Young slit doublet experiment, J. Opt. Soc. Am. B / Vol.26, No.12/ December 2009

[18] R.F. Egerton: Electron Energy-Loss Spectroscopy in the Electron Microscope, 2nd Edition, Plenum Press New York and London, 1996

[19] P.E. Batson: New surface plasmon resonance in clusters of small aluminum spheres, Ultramicroscopy 9 (1982) 277-282

[20] A. Liebsch: Electronic Excitations at Metal Surfaces, Physics of Solids and Liquids, Plenum Press, New York 1997

[21] P. Potapov, J. Verbeeck, P. Schattschneider, H. Lichte, D. v. Dyck: Inelastic electron holography as a variant of the Feynman thought experiment. Ultramicroscopy 107 (2007) 559-567.

\section{Acknowledgements}

Discussions at the Minisymposium on Inelastic Coherence and Holography, (5.-7. April 2009, Triebenberg Lab, TU Dresden) with

Christian Colliex (UPS), Claudie Mory (UPS), Lionel Calmels (CNRS Toulouse), Helmut Kohl (U Muenster), Harald Rose (TU Darmstadt), Peter Schattschneider (TU Wien), Jo Verbeeck (U Antwerp), Christoph Koch (MPI Stuttgart), Pete Nellist (U Oxford), Pavel Potapov (Globalfoundries, Dresden), Walter Strunz (TU Dresden) and Axel Lubk (TU Dresden) have been indispensable.

We are grateful to Dr. Petr. Formánek (Leibniz Institute of Polymer Research Dresden, Germany) for preparing the Al-specimen by FIB, and to Heide Müller and Bernd Einenkel for technical assistance.

The Francqui Foundation (Bruxelles) helped to establish the very fruitful cooperation of H.L. with the group of Dirk Van Dyck, $U$ Antwerp.

These investigations have been performed within European Union Framework 6 , Integrated Infrastructure, Reference 026019 ESTEEM. 